\title{
X-ray strain analysis in thin films enhanced by 2D detection
}

\author{
G. Geandier ${ }^{1}$, D. Faurie ${ }^{2, a}$, P.-O. Renault ${ }^{1}$, C. Le Bourlot ${ }^{2}$, P. Djemia ${ }^{2}$, O. Castelnau ${ }^{3}$, S. M. Chérif ${ }^{2}$, \\ E. Le Bourhis ${ }^{1}, \mathrm{Ph}$. Goudeau ${ }^{1}$ \\ ${ }^{1}$ Institut P' CNRS - Université de Poitiers - ENSMA, UPR3346, 86962 Futuroscope, France \\ ${ }^{2}$ LPMTM, UPR9001 CNRS, Université Paris13, 93430 Villetaneuse, France \\ ${ }^{3}$ PIMM, ENSAM, 151 Bd de l'Hopital, 75013 Paris, France
}

\begin{abstract}
Performing a complete in-situ strain measurement of polycrystalline thin films using X-ray diffraction is time consuming with most standard diffraction beamlines at synchrotron facilities and not realistic with laboratory diffractometers. Two dimensional detection is shown to enable relatively fast and reliable $\mathrm{X}$-ray strain measurements during continuous in-situ tensile testing of metallic films deposited on polyimide substrates. We show in this paper the advantages to perform this kind of measurements as compared to those with punctual detection.
\end{abstract}

\section{Introduction}

Both theoretical and experimental studies have shown that materials that have previously been examined at the micrometre scale must be re-examined at the nanometre scale in order to gain a full understanding of their physical properties. When grain sizes decrease to the nanometre scale, mechanical properties can be significantly altered compared with those of similar micrometre-sized grains [1]. The study of elastic behaviour consists in choosing a constitutive law using certain elastic constants. X-ray diffraction (XRD) is a well known technique used to measure very precisely elastic strains in crystalline materials [2]. As most crystalline materials are heterogeneous from the mechanical point of view, X-ray strain measurements have to be carried out for several directions, i.e. for different Bragg peaks or/and different lattice planes, to obtain reliable results. Both problems are rather sensitive to the quality of the experimental data and to the micromechanical models. Micromechanical models, such as the self-consistent model (SC), can predict inter-granular effects by accounting for the mechanical anisotropy and the texture of the polycrystal [3].

At high-flux synchrotron sources, the diffraction signal is much increased compared with conventional X-ray sources. Owing to the small diffracting volume in thin films and the necessity of performing the measurements in several directions for each applied load, the use of point detectors requires very long data collection times. Thus, to reduce the collection time a few load steps are performed. In order to obtain the necessary results for orientation studies and to accomplish the mechanical behaviour analysis in a reasonably cost-effective time frame, two-dimensional detectors are an appropriate solution [4]. High throughput of materials analysis without sacrificing data quality

a e-mail : faurie@univ-paris13.fr

This is an Open Access article distributed under the terms of the Creative Commons Attribution-Noncommercial License 3.0, which permits unrestricted use, distribution, and reproduction in any noncommercial medium, provided the original work is properly cited. 
is possible. Two-dimensional detectors provide simultaneous collection of diffraction data from several lattice planes, thus allowing a more complete assessment and understanding of the mechanical behaviour of crystalline thin films, in particular, a better knowledge of elastic grain interactions. Two-dimensional detectors could be helpful for the analysis of multiphased thin films, as the signals from all phases are recorded in a single exposure.

In this paper, we remind the "classical" geometry used for X-ray strain analysis during in-situ tensile testing. Afterwards, we present an acquisition strategy based on reflection-transmission geometry and achieved with synchrotron XRD with two-dimensional detectors. This strategy allows the optimization of the measurement time and increases the data quality and reliability for the study of mechanical behaviour of thin films. By collecting more complete data sets in a shorter period of time, orientation studies can be accomplished in a time frame that is acceptable to the experimentalist requiring several diffraction peaks and several applied loads.

\section{X-ray diffraction and in-situ tensile tests with punctual detection}

\subsection{Basics}

Elastic behaviour of supported thin films can be studied by coupling in-situ tensile tests and XRD. Intragranular strains are determined from the relative Bragg peak position shifts, while the overall stresses applied to the film-substrate composite are determined from a load cell fixed to one jaw of the micro-tensile tester [5-7]. For a purely elastic response, the local strain $\varepsilon_{\mathrm{ij}}(\mathbf{x})$ at any position $\mathbf{x}$ inside the material is given by

$$
\varepsilon_{\mathrm{ij}}(\mathbf{x})=\mathrm{S}_{\mathrm{ijkl}}(\mathbf{x})\left[\mathrm{B}_{\mathrm{klmn}}(\mathbf{x}) \bar{\sigma}_{\mathrm{mn}}\right]
$$

Where the stress localization tensor $\mathbf{B}$ can be estimated by means of full-fields or mean-fields homogenization methods. The relative shift of diffraction peaks position is measured with respect to line positions at the macroscopically unloaded state, since this allows for an accurate study of the mechanical behaviour without $a d$ hoc assumption regarding the residual stress distribution generated by the deposition process. The so-called "lattice strain" $\varepsilon_{\Phi, \Psi}^{\mathrm{hkl}}$ associated to the peak position shift is a direct measurement of the mean axial elastic strain

$$
\varepsilon_{\Phi, \Psi}^{\mathrm{hkl}}=\mathrm{n}_{\mathrm{i}} \mathrm{n}_{\mathrm{j}}<\varepsilon_{\mathrm{ij}}>_{\Omega}=\mathrm{n}_{\mathrm{i}} \mathrm{n}_{\mathrm{j}}\left[<\mathrm{S}_{\mathrm{ijkl}}(\mathbf{x}) \mathrm{B}_{\mathrm{klmn}}(\mathbf{x})>_{\Omega} \bar{\sigma}_{\mathrm{mn}}\right]
$$

with $\mathbf{n}$ a unit vector parallel to the scattering vector $\mathbf{q}$ (given by the diffraction angles $\Phi$ and $\Psi$, see Fig. 1), and $\langle.\rangle_{\Omega}$ denoting the average over the diffracting volume $\Omega$ associated to the $\{h k l\}$ diffracting plane [8].

The components $<\mathrm{S}_{\mathrm{ijkl}}(\mathrm{x}) \mathrm{B}_{\mathrm{klmn}}(\mathrm{x})>_{\Omega}$ provide general expressions for the X-ray elastic constants (XEC) [9]. Eq. (2) recovers the classical $\sin ^{2} \Psi$ law for macroscopically isotropic materials (linear relationship between lattice strain and $\sin ^{2} \Psi$ ) given here for a biaxial macroscopic stress state generally used for thin films:

$$
\varepsilon_{\Phi, \Psi}^{\mathrm{hkl}}=\frac{1}{2} S_{2}^{\mathrm{hkl}}\left(\bar{\sigma}_{11} \cos ^{2} \Phi+\bar{\sigma}_{22} \sin ^{2} \Phi\right) \cdot \sin ^{2} \Psi+\mathrm{S}_{1}^{\mathrm{hkl}}\left(\bar{\sigma}_{11}+\bar{\sigma}_{22}\right)
$$

Where $\frac{1}{2} S_{2}^{\text {hkl }}$ and $S_{1}^{\text {hkl }}$ are $h k l$-dependent XECs of macroscopically isotropic material [2].

Using a four-circle goniometer, it is possible to characterize the elastic behaviour for almost any orientation of $\mathbf{n}$ (see Fig. 1). Then, a least-square fitting procedure is adopted to adjust results of the SC model to experimental data and to determine single-crystal and subsequent macroscopic elastic constants, the crystallographic texture of the film being known. 


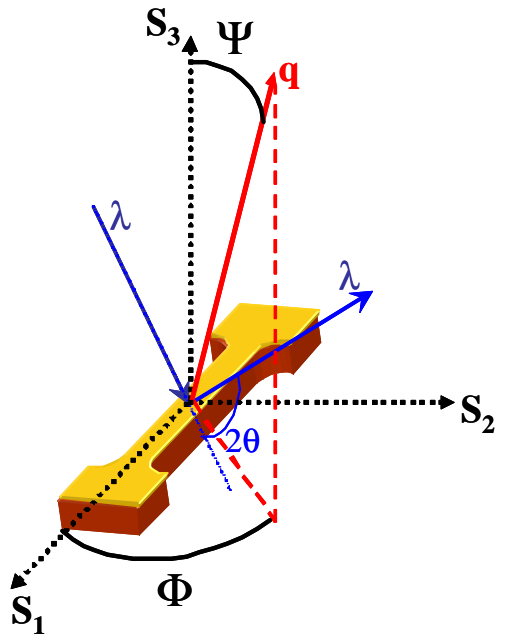

Fig. 1. Geometry of the diffraction setup used for the in-situ loaded 'dog bone' specimens. The uniaxial tensile stress applied to the film-substrate structure is along $S_{1}$. The direction of the scattering vector $\mathbf{q}$ is defined by the in-plane azimuthal angle $\Phi$ from $S_{1}$ and the polar angle $\Psi$ from the sample normal direction $S_{3}$.

\subsection{Results}

An example of XRD experiments for a $700 \mathrm{~nm}$ thick gold film with punctual detection is shown on Fig. 2. Experiments were performed at the LURE synchrotron radiation facility on beamline DW22. The tensile module was at the centre of a four-circle diffractometer. The beam size was set to $100 \times 500 \mu \mathrm{m}$, that is, much larger than the average in-plane grain size of the gold film (about 100 $\mathrm{nm}$ ). Strain measurements were made at $\Phi=0^{\circ}$ (longitudinal direction) and at different $\Psi$ positions (from $0^{\circ}$ to $75^{\circ}$ ) for different $\{h k l\}$ planes. Overview of strain values according to the diffraction condition as a function of load is shown on Fig. 2. We observe that all strain values increase linearly with load as expected for linear elasticity. Obviously, strain is either compressive (negative) or tensile (positive). Moreover, the experimental strains can be well reproduced by SC model, as described in Faurie et al. [8].

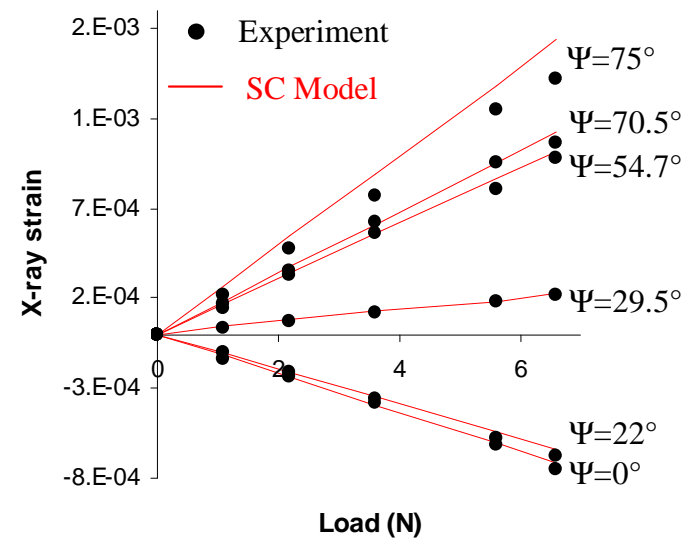

Fig. 2. Overview of experimental and calculated strain values according to the diffraction condition as a function of load.

Even if these experiments were very precise, they were also time-consuming (about 2 days), that is not reasonable for achieving a study of several films with different microstructures. 


\section{X-ray diffraction and in-situ tensile tests with 2D detection}

\subsection{Technical aspects}

X-ray experiments combining both reflection and transmission modes were carried out at D2AM beamline (ESRF), as described in Geandier et al. [10]. This mode is called reflection-transmission mode, as one part of the signal is also studied in transmission thanks to the X-ray transparency of the substrate. In this geometry, strain information is obtained for a given set of direction, i.e. a given set of $(\Phi, \Psi)$ positions, where $\Phi$ and $\Psi$ are the so-called diffraction angle. With a single exposure, this acquisition strategy suffices to perform strain measurements in the three-dimensional space.

The samples were mounted with an angle $\omega$ (angle between the sample surface and the incident beam direction) of $14^{\circ}$ or $18^{\circ}$ (dependent of studied films) (Fig. 3) with an energy of $20 \mathrm{keV}$. No absorption correction has been added as we are measuring the relative shift of the Bragg peaks via the shift of the centre of the fitted function. The distance between the sample and the detector was set to $123 \mathrm{~mm}$, which corresponds to the minimum distance available in the presence of the beam stop. Moreover, Si powder was placed on the backside of the sample, close to the measuring point, in order to correct for the sample drift during loading. We used Si powder because it offers a sufficient number of diffraction rings at angles covering the whole detector surface. Diffraction patterns on the Si powder were taken at each load step.

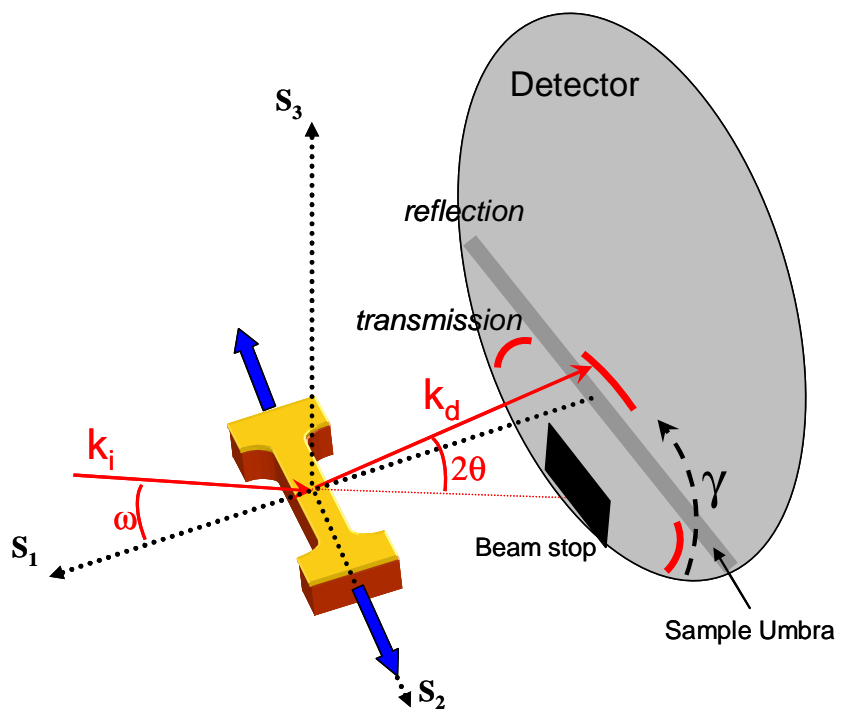

Fig. 3. Setup used to record the diffractogram patterns during the in-situ tensile tests for the reflectiontransmission mode on the D2AM beamline at ESRF.

Because of the geometry of the experiment and the transparency of the substrate to X-rays, we catch two types of signals on the CCD images. The diffraction patterns (Fig. 4) show partial Debye rings, which are continuous for the Si powder and with intensity variations for the Ni thin film (because of the fibre texture). The right part of the image shows the reflected signal from the sample, while the left part shows the transmitted signal. As the silicon powder signal and metallic films signal are recorded at the same time, Si powder rings were used to calibrate the geometrical parameters of the experiment, assuming that the wavelength of the X-ray beam remains stable. 
Using fit2d (ESRF software [11]) on silicon rings, the sample-to-detector distance as well as the image centre and the detector non-orthogonality were calibrated. We have already noted that the distance may change for the first load steps. When the applied force reaches a few Newtons, the distance remains stable until the end of the experiment. Using the calibrated geometry parameters (centre, distance and tilt angle) the data were distributed into $1^{\circ} \delta \gamma$ bins and integrated azimuthally in a process referred to as 'caking' in the fit $2 \mathrm{~d}$ lexicon, where $\gamma$ is the angle describing the Debye rings (a combination of $\Phi$ and $\Psi$, see Fig. 3). Fig. 5 shows an image at this step of the procedure. The reduced data were saved to obtain, for each load step, the classical $2 \theta$-intensity diagram for each bin, which contains 166 diagrams per load step.

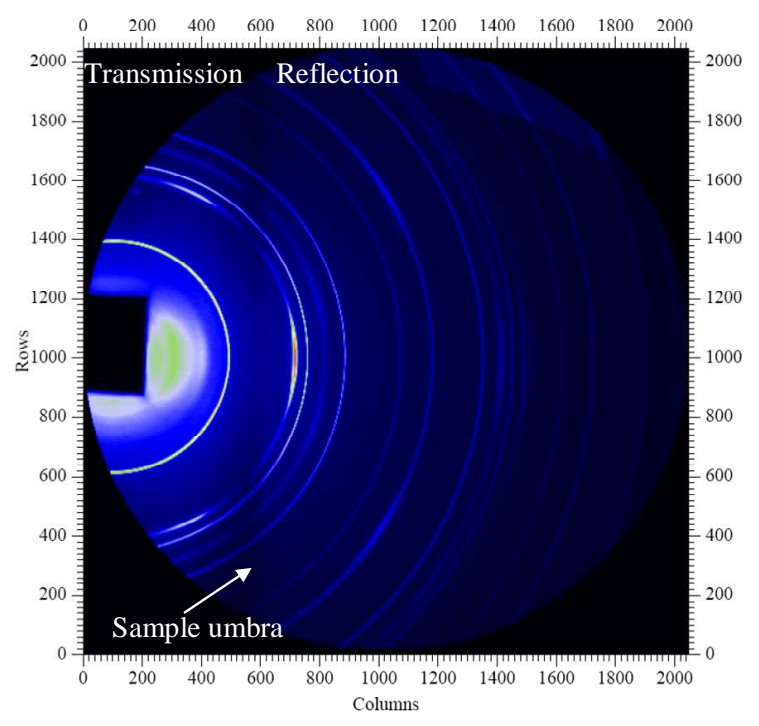

Fig. 4. Whole diffraction pattern in the reflection-transmission geometry. The figure shows Ni peak details in the reflection and transmission parts of the image. The sample umbra allows the distinction between the two parts.

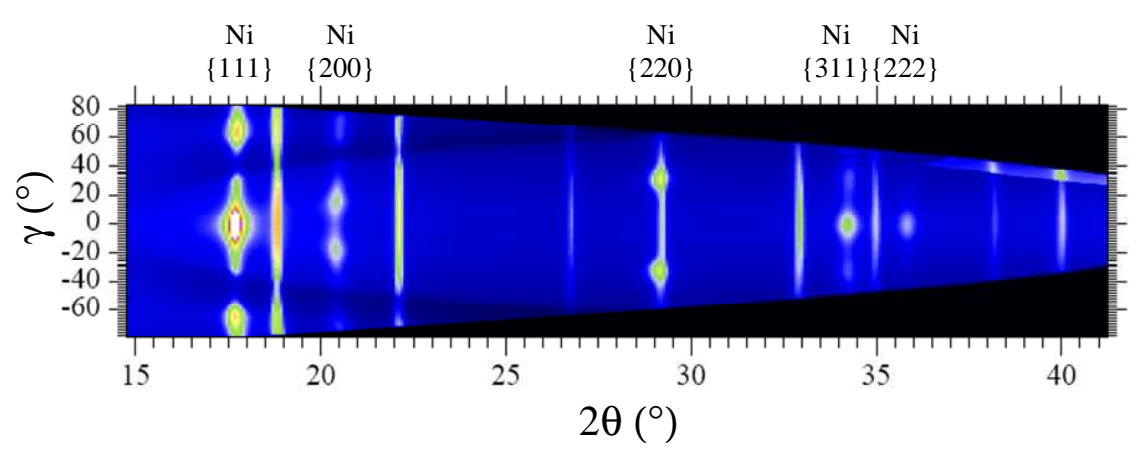

Fig. 5. Image of the data reduced by fit $2 \mathrm{~d}(2 \theta$-intensity) in the transmission geometry for a $200 \mathrm{~nm}$ thick Ni films. It is clear here that intensity varies with $\gamma$ because of $\{111\}$ fibre texture of Nickel films. 


\subsection{Experimental Results}

Another in-house program was used to generate inputs for gnuplot in order to fit the Si peaks that are complete over the ring and $\{111\} \mathrm{Ni}$ peaks. The code allows us to localize the peak and to extract its parameters, so that the adjustment procedure in gnuplot using a Pearson VII function starts with parameters close to the final solution. Keeping $\gamma$ constant, parameters from a previous adjustment are passed to the next load step in the gnuplot script. The parameters extracted from each peak adjustment are the position, intensity, FWHM, shape factor and background polynomial forms (which can be of degrees 0-6). At the end of this procedure, all the parameters for the metallic films and $\mathrm{Si}$ peaks are collected for each load step and for each $\gamma$ position (166 in total).

Fig. 6 shows the example of experimental data for a $200 \mathrm{~nm}$ thick Nickel film. The elastic deformation for the $\{111\}$ reflection is plotted as a function of applied load, for 5 different $\gamma$ positions. The response is linear, as expected for linear elasticity, and the magnitude depends on $\gamma$ positions. Since the elastic fields are complexes, deformation can be positive or negative, depending on the measurement direction. The positions $\gamma=0^{\circ}$ and $\gamma=44^{\circ}$ correspond respectively to the strongest and the weakest nickel peak intensity for the $\{111\}$ reflection. This is the reason why the dispersion for $\gamma=44^{\circ}$ is relatively high as compared to other positions. The whole experimental results will be compared to micro-mechanical models as in Faurie et al. [8]. Moreover, we have planed to study evolution of magnetic properties of Ni films during tensile tests, by using Brillouin Light Scattering (BLS) combined in-situ with the micro-tensile tester, and thus to compare BLS results with the XRD ones to extract magneto-elastic coefficients. To conclude, performing a complete strain analysis of thin films using XRD is time consuming with most standard beamlines at synchrotron facilities and not realistic with laboratory diffractometers. Two-dimensional detection is shown to enable relatively fast (a few hours) and reliable X-ray strain measurements during in-situ tensile testing of thin films deposited on polyimide substrates.

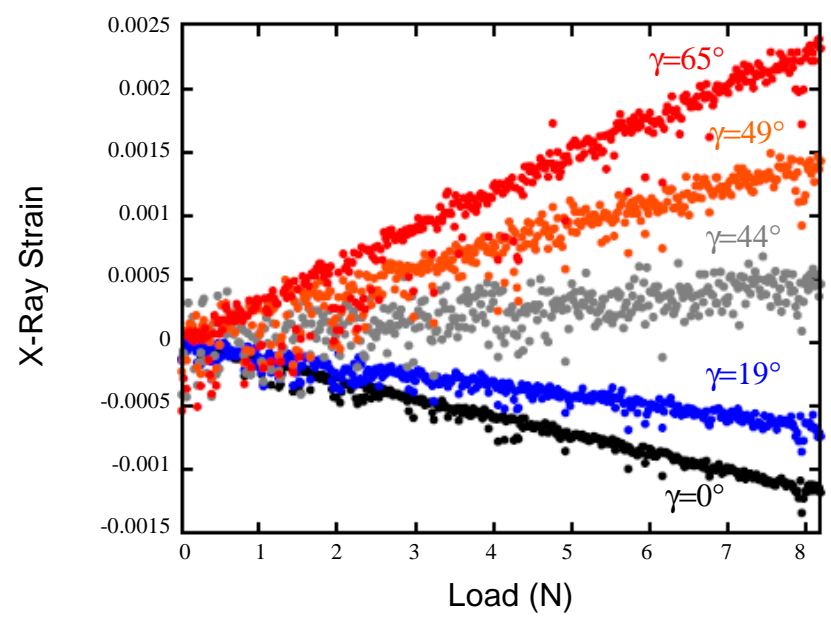

Fig. 6. Deformation measured by XRD as a function of applied load, for 5 different $\gamma$ positions, for the $\{111\}$ Bragg reflection. 


\section{Acknowledgements}

The authors would like to thank the D2AM beamline technical staff (European Synchrotron Radiation Facility (ESRF), Grenoble).

\section{References}

1. F. Spaepen, D. Y. W. Yu, Scr. Mater., 50, 729-732 (2004).

2. V. Hauk. Structural and Residual Stress Analysis by Non Destructive Methods: Evaluation, Application, Assessment. Amsterdam: Elsevier Science (1997).

3. S. Matthies, H. G. Priesmeyer, M. R. Daymond, J. Appl. Cryst., 34, 585-601 (2001).

4. G. Geandier, P.-O. Renault, E. Le Bourhis, Ph. Goudeau, D. Faurie, C. Le Bourlot, Ph. Djémia, O. Castelnau, S. M. Chérif, Appl. Phys. Letters, 96, 041905 (2010)

5. P.-O. Renault, E. Le Bourhis, P. Villain, Ph.Goudeau, K. F. Badawi, D. Faurie, Appl. Phys. Lett., 83, 473 (2003).

6. D. Faurie, P.-O. Renault, E. Le Bourhis, Ph. Goudeau, O. Castelnau, R. Brenner, G. Patriarche, Appl. Phys. Lett., 89, 061911 (2006).

7. D. Faurie, P.-O. Renault, E. Le Bourhis, Ph. Goudeau, Acta Mater., 54, 4503-4513 (2006).

8. D. Faurie, O. Castelnau, R. Brenner, P.-O. Renault, E. Le Bourhis, P. Goudeau, J. of Appl. Cryst., 42, 1073-1084 (2009)

9. N. Letouzé, R. Brenner, O. Castelnau, J. L. Bechade, Scr. Mater., 47, 595-599 (2002).

10. G. Geandier, P.-O. Renault, S. Teat, E. Le Bourhis, B. Lamongie, Ph. Goudeau, J. Appl. Cryst., 41, 1076-1088 (2008).

11. A.P. Hammersley, S.O. Svensson, A. Thompson, Nuclear Inst. and Methods in Physics Research, A 346, 312-321 (1994) 